\title{
LOS BRIÓFITOS DEL HERBARIO DE WILLKOMM (COI). I. HEPATICAE
}

\author{
M.Carmen VIERA y Juan REINOSO
}

\begin{abstract}
RESUMEN. Los briófitos del Herbario de Willkomm (COI). I. Hepaticae. En este trabajo se hace la revisión de los briófitos, en lo que concierne a las hepáticas, del herbario de Willkomm. Se trata de 25 táxones que se encuentran en la carpeta número 5 de dicho herbario y de los que hasta el momento no se tenia noticia de su existencia.
\end{abstract}

Palabras clave. Herbario, Willkomm, Briófitos, Hepáticas.

RESUMÉ. Les bryophytes de l'Herbier Willkomm (COI). I. Hepaticae. Dans ce travail on fait la revision des bryophytes en ce qui concerne a des hépatiques dans l'herbier de Willkomm, à ce moment considereès inédits. Il s'agit de 25 taxons qui se trouvent dans la carpette numéro 5.

Mots clés. Herbier, Willkomm, Bryophytes, Hépatiques.

ABSTRACT. The bryophyts of Herbar Willkomm (COI). I. Hepaticae. In the present work a revision of bryophyts of herbar Willkomm (COI) is presented. Twenty five taxa in the box number five, at the present unknown, are recognized.

Key words. Herbar, Willkomm, Bryophyts, Liverworts.

\section{INTRODUCCIÓN}

Con este trabajo pretendemos contribuir a lo que creemos es una labor básica de cara a dar a conocer colecciones antiguas consideradas inéditas y rescatadas de herbarios generales. Tal es el caso de la colección de briófitos del herbario de Willkomm (COI).

Para ello, durante los años 1990 y 1991 en diferentes estancias en el Instituto Botânico Dr. Julio Henriques da Universidade de Coimbra (Portugal)», hemos llevado a cabo la catalogación y revisión de los briófitos del herbario de Willkomm (Herbarium

Realizado en el marco del Proyecto Nr.3747/91 subvencionado por el Vicerrectorado de Investigación de la Universidad de Santiago de Compostela 
mediterraneum, pyrenaicum et canariense), en depósito en el COI. Dichos briófitos están contenidos en dos carpetas, la número 4 (Musci Frondosi) y la número 5 (Hepaticae).

En este primer trabajo hemos revisado todo el material de hepáticas contenido en la carpeta número 5. Después de efectuar la revisión se han contabilizado 25 hepáticas. Queremos hacer constar que en dicha carpeta, junto al material briofítico, existen también algunos pliegos de algas.

Gran parte de las recolecciones fueron efectuadas por el propio Willkomm como resultado de los viajes realizados por la Península Ibérica Willkomm , ( 1844a,b; 1845a, b; 1846 y 1850). Durante este tiempo visita: Valencia (Sagunto, Cerro de Santa María en la Sierra de Chiva, etc.), Granada (Sierra Nevada, la Alhambra y el Generalife), Gibraltar, Málaga, Irún y el Algarbe (Sierra de Monchique) en Portugal. De regreso a su país, vuelve finalmente a España en 1873, año en que visita las islas Baleares.

En la colección de briófitos del herbario de Willkomm hay que resaltar las aportaciones realizadas por el botánico español Loscos y los extranjeros: Rossmaessler y Verdoorn . Estos y otros botánicos contemporáneos se dirigían a Willkomm no sólo para intercambio de duplicados, sino también, en la mayor parte de los casos, para resolver problemas referentes a la identificación de plantas e información y obtención de bibliografía. No en vano Willkomm figuraba entre los botánicos que gozaban de mayor prestigio a nivel mundial y quien mejor conocía la flora de la Península Ibérica, pues era autor, junto con J. Lange, del «Prodromus Florae Hispaniae», la flora más completa de la Península Ibérica que existe.

Es interesante señalar también la existencia en el herbario de pliegos procedentes de otras colecciones: Soleirol (Herb. Cors.), F. Schultz (Herbarium normale), Bourgeau (Pl. Canarienses), Huet du Pavillon (Plantae Siculae) y Marcucci.
De toda la colección resalta por lo cuidadoso y minuciosamente etiquetado, al igual que por su buen estado de conservación, el material recolectado y determinado por el propio Willkomm .

En otros casos los ejemplares se encontraban sueltos fuera de los pliegos, desmenuzados y bastante deteriorados. De manera que hemos procedido a su debida restauración manteniendo escrupulosamente todas las anotaciones y etiquetas de los diferentes autores y recolectores. También la mayoría de los ejemplares llevan etiquetas con anotaciones manuscritas de Willkomm así como de otros autores, tal es el caso de Loscos.

La mayor parte de las especies estaban determinadas, en gran medida buenas determinaciones, en otras se ha procedido a su identificación y en todos los casos se han actualizado los diferentes táxones, manteniendo como queda dicho las anotaciones originales.

Para la identificación, nomenclatura y ordenación de los táxones en el catálogo, se han seguido los trabajos de: Bonner (19621978), Casares Gil (1919), Dull (1983), Grolle (1976,1981 y 1983a, b), Husnot (1922), JovetAst (1983 y 1986), Macvicar (1971), Müller (1906-16), Schuster (1969 y 1983), Smith (1990), Stefani (1906-1917), entre otros.

Para cada taxon se indica nombre correcto, sinónimo de interés, nombre con el que aparece en el pliego y etiqueta (s) que acompaña al (los) ejemplar (res).

Para la transcripción de las etiquetas hemos adecuado el trabajo a las normas habituales de transcripción (Candollea, 36: 543-584, 1981). Las alusiones a las letras se abrevian como [m], así « (letra manuscrita de Willkomm)» queda como «[m. Willkomm]»; «(letra manuscrita de ?)» queda como «[m.i.]» la abreviatura habitual para «manu ignota» cuando es imposible descifrar el autor que ha escrito sobre las etiquetas. Las etiquetas impresas se señalan como [impreso]. 


\section{HEPÁTICAS EN EL HERBARIO DE WILLKOMM}

1) Phaeoceros bulbiculosus (Brot.) Prosk., Rapp. Comment. VIII Congr. Int. Bot. 14/16:69 (1945) . (= Anthoceros bulbiculosus Brot., Fl. Lusit. 2:430 (1804)).

a) Anthoceros polymorpha Raddi

Etiqueta.- [impreso]: soleirol, herb. cors. // [m.i.]: 5040. Anthoceros polymorpha, Raddi/Calvi15 fevrier. 1

2) Sphaerocarpos michelii Bellardi, Appendix ad Fl. Piedmont. :52. Turin (1792). Mémoirs Acad. Roy. Sci. Turin 5:258 (1792)

a) Sphaerocarpus terrestris Micheli

Etiqueta.- [impreso]: F. Schultz, herbarium normale. Cent. 10. // 1000 bis, Sphaerocarpus terrestris Micheli. / Mars 1857. / Sur la terre une dans les allées du jardin botanique de Pise (Toscane, Italie). / Rec. P. Savi. /

\section{3) Targionia hypophylla L., Sp. Pl. 1136 (1753)}

a) Targionia hypophylla $L$.

Etiqueta.- [impreso]: Herbarium Willkommii. / [m. Willkomm]: Targionia hypophylla L. / Prope Malaga ad et Palo. / [impreso]: Legit [m. Willkomm]: Kalina d. 14/10 [impreso] 18 [m. Willkomm]: 59. I

b) T. Michelii Corda

Etiqueta 1.- [m. Willkomm]: Targionia Michelii Corda. / Hab. in Sierra de Yunquera in cavis rupium loco el Canoón, / ad alt. circ. 4500 5000'. / Legi d. 27 April 1845. /

Etiqueta 2.- [m. Willkomm]: Targionia Michelii Corda. / Crypt. exsicc. n. 60. (955): / Hab. in muris urbis Gades, praecipue in latere / septentrionali, copiose. / Legi mense Januaris 1845. I

Etiqueta 3.- [m. Willkomm]: Targionia Michelii Corda. / Crypt. exsicc. N. 60 (955). / Hab. in rupestribus humidis collinum las Lomas del Alcornoque prope oppidum / Medina-Sidonia. / Alt. 1000'. / Legi d. 22 Febr. 1845. /

4) Reboulia hemisphaerica (L.) Raddi, Opusc. Sci. (Bologna) 2: 357 (1818). (= Marchantia hemisphaerica L., Sp. Pl. 1138 (1753)).

a) Rebouillia hemisphaerica

Etiqueta 1.- [m. Willkomm]: (Herb. balear. n.
503) / [impreso]: Herbarium Willkomm. / [m. Willkomm]: Rebouillia hemisphaerica / Mallorca: in solo humido in parte superiore regionis / quercetorum in latere orientali mont. Peña d'en Galilén. / [impreso]: Legi [m. Willkomm]: d. 29 Apr. [impreso]: 18 [m. Willkomm]: $73 . /$

Etiqueta 2.- [m. Willkomm]: (Herb. balear n. 503.) / [impreso]: Herbarium Willkomm. / [m. Willkomm]: Rebouillia hemisphaerica / Mallorca: prope vall. N.S. de LLura ad fontana / publicum. / [impreso]: Legi [m. Willkomm]: d. 28 Apr. [impreso]: 18 [m. Willkomm]: $73 . /$

Etiqueta 3.- [m. Willkomm]: Rebouillia hemisphaerica Raddi. / Hab. in muris humidis theatri antiqui/Saguntini prope oppidum Murviedro / regni Valentini. / Legi d. 11 Maji 1844. /

Etiqueta 4.- [m. Willkomm]: Rebouillia hemisphaerica Raddi. / Hab. in latere occidentali montis / Gibraltarici in cavis fissurique rupium / com Marchantia paleacea et Targionia / Michelii. / legi d. 1 April 1845. I

Etiqueta 5.- [m. Willkomm]: Rebouillia hemisphaerica Raddi / Murcia [m.i] / Leg. Rossmaessler. /

5) Lunularia cruciata (L.) Lindb., Bull. Soc. Roy. Bot. Bel. 13: 147 (1874). (=Marchantia cruciata L., Sp. Pl. 1137 (1753))

a) Lunularia vulgaris Mich.

Etiqueta 1.- [m. Willkomm]: Lunularia vulgaris. Mich. / Plant. hisp. exsicc. Crypt. $n^{\circ}$ 61. / (953) / [m.i.] Alhambra [m.i.] Fuente de Avellanas / [m.i.] Targionia Michelii [m.i.] / Leg. am. 29 Obtob. 1844 /

Etiqueta 2.- [m. Willkomm]: Marchantia paleacea Bertol. / Hab. in latere occidentali / montis Gibraltarisci. / Legi d. 1 Apr. 1845. I

Etiqueta 3.- [m. Willkomm]: Marchantia paleacea Bertol. / Hab. in collibus Las Lomas del Alcorconque / prope Medina-Sidonia. / Legi d. 22 Feb. $1845 /$

6) Preissia quadrata (Scop.) Nees, Naturg. Europ. Leberm. 4: 135 (1838). (= Marchantia quadrata Scop., Fl. Carniol. ed. 2. 2:355 tab. 63. (1772).

a) Targionia Michelii Corda

Etiqueta.- [m. Willkomm]: Targionia Michelii. Corda / Crypt. exsicc. No60/(955)/ Hab. in rupestribus humidis collinum las Lomas del Alcornoque prope oppidum / Medina-Sidonia. / 
Alt. 1000'. / Legi d. 22 Febr. 1845. /

7) Marchantia polymorpha L., Sp. Pl., ed. 1, 2: 1137 (1753)

a) Marchantia polymorpha L. B. alpestris N. ab E., B. riparia. N. ab. E.

Etiqueta.- [m. Willkomm]: Marchantia polymorpha. L. (v. scyph.) / B. alpestris. N. ab E. / B. riparia N. ab E. / Hab. in Sierra Nevada ad river in parte superiore vallis Fluvii Monachil / passim. / Alt. 6 -7000'. / Leg. d. 3 Aug. 1844. /

8) Marchantia paleacea Bertol., Opusc. Sci. (Bologna) 1: 242 (1817)

a) Marchantia paleacea Bertol.

Etiqueta 1.- [m. Willkomm]: Marchantia paleacea Bertol. / Crypt. hisp. exsicc. $\mathrm{N}^{\circ} 1$. (954). / [m.i.] Alhambra / im Darrolfila, und an [m.i.] Alhambra [m.i.] / Legi d. 8 Oct. 1844. /

Etiqueta 2.- [m. Willkomm]: Marchantia paleacea Bertol. / Crypt. hisp. exsicc. No 1. (954) / [m.i.] von Sagunt bai Murviedro / im [m.i.] Valencia. / Leg. d. 11 Mai 1844. /

Etiqueta 3.- [m. Willkomm]: Marchantia paleacea Bertol. / Crypt. hisp. exsicc. $N^{\circ} 1$. (954) / [m.i.] Alhambra und dam / Generalife [m.i.] Alhambra ? / Leg. d. 28 Oct. 1844.

9) Oxymitra paleacea Bisch. in Lindb., Nova Acta Phys. Med. Acad. Caes. Leop. Carol. Nat. Cur. 14: 124 (1829)

a) Riccia pyramidata Raddi

Etiqueta.- [impreso]: soleirol, herb. cors. // [m.i.]: 5004. Riccia pyramidata, Raddi / Cabri. / 20 Septembre./

10) Riccia ciliifera Link ex Lindenb., Nova Acta Phys. Med. Acad. Caes. Leop. Carol. Nat. Cur. 14: 119 (1829)

a) Marchantia

Etiqueta.- [m. Willkomm]: Marchantia/Hab. in rupestribus collinum Las Lomas del Alcornoque prope oppidum / Medina-Sidonia. / Alt. 1000'. / Legi. d. 22 Febr. 1845. I

11) Riccia lamellosa Raddi, Opusc. Sci. (Bologna) 1: 351 (1818)

Etiqueta .-[impreso]: PLANTAE SICULAE/ / Riccia lamellosa Raddi. / In argillosis maritimis hieme inundatis / propè Ficarazi. / 8 Apr. 1855.
Leg. E. et A. Huet du Pavillon. /

a) Riccia favosa Willk.

Etiqueta .- [m. Willkomm]: Riccia favosa Willk. / Hab. in Sierra Nevada in arena humida calcarea / ad fluvium Monachil inter villas Cortijo de / Echa y de S. Gerónimo rarissime ! 4500'. / Leg. d. 10 Octob. 1844.

b) R.Dufourii N. ab E.

Etiqueta .- [m. Loscos]: Riccia Dufourii N. ab E. / Aragon. Leg. Loscos

12) Metzgeria furcata (L.) Dum., Recueil Observ. Jung. 26 (1835). (= Jungermannia furcata L., Sp. Pl. 1136 (1753))

a) Metzgeria furcata Nees

Etiqueta 1.- [impreso]: II. Metzgeria furcata Nees / mit Frullania dilatata símbolo masculino y femenino / Tracquitara auf Taxus baccata, Mag. / Un itin. crypt. 1866. Dr. Marcucci. /

Etiqueta 2.- [m. Willkomm]: Metzgeria furcata N. ab E. / Irun. / Legi Majo 1850. /

13) Aneura pinguis (L.) Dum., Sylloge Jung. Europ. Indic. 86 (1831). (= Jungermannia pinguis L. Sp. Pl. 1136 (1753)).

a) Aneura pinguis Lam. var.fascialis Hampe.

Etiqueta.- [m. Willkomm]: Aneura pinguis Dum. / var. fascialis Hampe / Crypt. hisp. exsicc. n. 40. (952). / Hab. in Sierra Nevada ad rivios regionis / montanae superiorisad fluvium Monachil / infra S. Gerónimo / Alt. 4800 ‘. / Legi d. 7 Aug. 1844. /

14) Pellia epiphylla (L.) Corda in Opiz, Beiträge Naturg. 12:654 (1829). (=Jungermannia epiphylla L., Sp. Pl. 1135 (1753)).

a) Pellia epiphylla N. ab E. A.B. speciosa

Etiqueta .- [m. Willkomm]: Pellia epiphylla N. ab E. / A.B. speciosa / Crypt. hisp. exsicc. $n^{\circ} .15$ (951). / Hab. in Sierra Nevada in rivo e cacumini Dornajo venicute / prope San Gerónimo, ad alt. c. 5000'. / Legi d. 29 Julio 1844. I

b) Pellia epiphylla N.ab E. / A.B.* speciosa longifolia.

Etiqueta .- [m. Willkomm]: Pellia epiphylla N. ab E. / A.B. * speciosa longifolia. / Hab. in regni Valentini regione subalpina: in Sierra/de Chiva in fonte Fuente de Boquiva in valleculas que / ad cacumen Santa Maria ad adfrenditur./ Alt. c. 5000'. / Legi d. 2 Junii 1844. /

c) Pellia epiphylla N. ab E. A. alfa. fertilis. 
Etiqueta 1.- [m. Willkomm]: Pellia epiphylla N. ab E. / A. alfa fertilis. (abarque sporangiis) / Hab. in Sierra Nevada in cavis madidis ad rivum vallis barranco de Castillejos / Alt. c. 4000'. / Legi d. 21 Aug. 1844./

Etiqueta 2.- [m. Willkomm]: Pellia epiphylla N. ab E. / A. alfa fertilis. (Abarque sporang.) / Crypt. exsicc. 15. (951). / Hab. in regione alpina montis Sierra de Chiva / regni Valentini in cavis humidis rupium calcarearum/orientem spectrantum cacuminis Cerro de Santa Maria / Alt. c. 5800'. / Legi d. 2 Junnii 1844. I

d) Pellia epiphylla N. ab E. B. delta crispa

Etiqueta.- [m. Willkomm]: Pellia epiphylla N. ab E. / B. delta crispa. / Crypt. hisp. exsicc. 59. (951). / Hab. prope urbem Granada/ad aquaeductum arcis Alambra. / Alt. 2500'/ Legi d. 8 Octb. 1844 ./

e) Pellia epiphylla N. ab E. B. E. undulata. forma

prolifer.

Etiqueta.- [m. Willkomm]: Pellia epiphylla N. ab E. / B.E. undulata. forma prolifera. / Crypt. hisp. exsicc. n. 59 (950) / Hab. prope urbem Granada in cavis madidis / inter fontes Fuente de Avellanas et Fuente la Grila. / Alt. 2500'. / Legi d. 8 Oct. 1844. I

15) Jungermannia exsertifolia Steph. subsp.cordifolia (Dum.) Vána Folia Geobot. Phytotax. 8: 268 (1973). (= Haplozia cordifolia Dumort., Bull. Soc. Roy. Belgique 13:59 (1874).

a) Jungermannia cordifolia Hook. var. laxa Hampe

Etiqueta.- [m. Willkomm]: Jungermannia cordifolia Hook. / var. laxa Hampe. / Crypt. hisp. exsicc. 38 (947). / Hab. in Sierra Nevada in rivulis pratorum alpinorum: / in parte superiore vallis fluvii Monachil et in Borreguil / de S. Gerónimo ad alt. circ. 6 -8000'. / Legi d. 3 Aug. 1844. /

16) Jungermannia atrovirens Dum., Sylloge Jung . Europ. Indig: 51 (1831)

a) Jungermannia riparia Tayl.

Etiqueta.- [impreso]: III. [m.i.] Jungermannia riparia Tayl. I Tacpuitara (Barbargia), sommersa nelle acque calcarifere, Mag./Un. itin. crypt. 1866. Dr. Marcucci. I

17) Plagiochila spinulosa (Dicks.) Dum., Recueil Observ. Jung.: 15 (1835). (= Jungermannia spinulosa Dicks., Fasc. Sec. Pl. Crypt. Brit.: 14 (1790)).

a) Plagiochila spinulosa Nees et Mont.

Etiqueta.- [impreso]: E. Bourgeau, PL. CANARIENSES (ex itinere secundo) 1855.//1600. PLAGIOCHILA SPINULOSA Nees et Mont..Phyt. Can. IV, 46. / (Montagne.) / Teneriffa: in sylva Las Mercedes, a la Cumbre, ad rivulos. / 10 februar.

18) Lophocolea bidentata (L.) Dum., Recueil Observ. Jung. 17 (1835). (= Jungermannia bidentata L. Sp. Pl. 1132 (1753)).

a) Lophocolea bidentata N. ab E.

Etiqueta.- [m. Willkomm]: Lophocolea bidentata / N. ab E. / Irun. /

19) Chiloscyphus polyanthos (L.) Corda in Opiz, Beitr. Naturg. 12:651 (1829). (= Jungermannia polyanthos L., Sp. Pl. 1131 (1753)).

a) Chiloscyphus polyanthus Corda. var. rivularis $\mathrm{N}$. ab E.

Etiqueta 1.- [m. Willkomm]: Chiloscyphus polyanthos Corda. / b. rivularis N. ab E. / Hab. in Sierra Nevada ad rivulus in parte / superiore vallis Fluvii Monachil cum Marchantia / polymorpha . / Alt. 6-7000'. / Legi d. 3 Aug. 1844. I

Etiqueta 2.- [m. Willkomm]: Chiloscyphus polyanthos Corda. / 21 / B. rivularis. /

b) Chiloscyphus polyanthus Corda.

Etiqueta.- [m. Willkomm]: Chiloscyphus polyanthos Corda. / Crypt. hisp. exsicc. n. 37 (948). / Hab. in Sierra Nevada in rivulis regionis alpinae superioris: en el Borreguil / de San Gerónimo ad alt. circ. 7-8000'. / Legi d. 3 Aug. 1844. /

20) Scapania compacta (A. Roth) Dum., Recueil Observ. Jung. 14 (1835). (= Jungermannia compacta Roth, Tent. Flor .Germ. 3: 375 (1799)).

a) Jungermannia inflata Huds.

Etiqueta.- [impreso]: E. Bourgeau, PL. CANARIENSES (ex itinere secundo) 1855.//1592. JUNGERMANNIA INFLATA Huds. - Phyt. Can. IV, 49. / (Montagne.) / Teneriffa: Tegueste el Viejo, ad terram in umbrosis. 7 februar. I

21) Scapania nemorea (L.) Grolle, Rev. Bryol. Lichénol. 32: 160 (1963). (=Jungermannia nemorea L., Syst. Nat. ed. 10: 1337 (1759)).

a) Scapania nemorosa Nees. 
Etiqueta.- [impreso]: E. Bourgeau, PL. CANARIENSES (ex itinere secundo) 1855.//1593. SCAPANIA NEMOROSA Nees. / (Montagne.) / Teneriffa: Selva de las Mercedes, in udosis. 10 februar. $/$

22) Porella arboris-vitae (With.) Grolle, Trans. Br. Bryol. Soc. 5:770 (1969). (= Jungermannia arboris-vitae With., Bot. Arrang. All. Veget. Great Brit. 2: 697 (1776)).

a) Madotheca platyphylla Dumort.

Etiqueta.- [m. Loscos]: 53 Jungerman/nia tamarisci ?/[m. Willkomm]: Aragonia/Leg. Loscos./ = Madotheca platyphylla Dumort./

23) Porella platyphylla (L.) Pleiff., $F l$. Niederhessen und München 2:234 (1835). (= Jungermannia platyphylla L., Sp. Pl. 1135 (1753)).

a) Jungermannia laevigata Schrad.

Etiqueta.- [impreso]: Soleirol, herb. cors // [m. Willkomm]: 5011 Jungermannia laevigata, Schrad./Forêt du Pertico.

b) Madotheca platyphylla Dum.

Etiqueta 1.- [impreso]: Herbarium Willkomm. / [m. Willkomm]: Madotheca platyphylla Dum./ Aragonia australis/[impreso] : Legit, [m. Willkomm]: Loscos/[impreso]: 18

Etiqueta 2.-[m. Willkomm]: Madotheca platyphylla Dumort. Crypt. Hisp. exsicc. 14. (950)/ Hab. in region alpina regni Valentini: in Sierra de Chiva in rupibus spectantibus cacuminis / Cerro de Santa Maria, inter Saxifragam paniculatum Cav. caespiter/ densissimas formans./Alt. 5800!/Legi d.2 Junii/1844.

c) Jungermannia platyphylloidea Onés

Etiqueta.- [impreso]: Soleirol, herb. cors. // [impreso]: 5011 a. Jungermannia platyphylloidea, Onés,/Mont. Terrible, (Bologne)./

d) Madotheca laevigata Dumort.

Etiqueta.- [impreso]: E. Bourgeau, PL. CANARIENSES (ex itinere secundo) 1855./-/ 1581. Madotheca laevigata Dumort.- Phyt. Can. IV, 52./ (Montagne)/Teneriffa: Selva de las Mercedes, in petrosis. $10 \mathrm{febr}$.

24) Frullania tamarisci (L.) Dum., Rec. Obs. Jungerm. Tournay :13 (1835). (= Jungermannia tamarisci L., Sp. Pl.: 1134 (1753)).

a) Frullania

Etiqueta .- [impreso]: Herbarium Willkomm.
/ [m. Willkomm]: Frullania / Serra de Monchique in Algarbiis in / camino Foia / [impreso]: Legi [m. Willkomm]: Febr. [impreso]: 18 [m. Willkomm]: 46. 1

b) Frullania tamarisci (L.) Dum.

Etiqueta .- [impreso]: HERB. [m. Verdoorn]: Inst. Bot. Coimbra / (Willkomm).- /[impreso]: $\mathrm{N}^{\circ} /[\mathrm{m}$. Verdoorn]: Frullania Tamarisci (L.) Dum. /3. 1929./ [impreso]: det. Verdoorn /

25) Frullania dilatata (L.) Dum., Rec. d'obs. Junger. 13 (1835). (=Jungermannia dilatata L., Sp. Pl. 1135 (1753)).

a) Frullania dilatata N. ab E. B. microphylla Etiqueta.- [m. Willkomm]: Frullania dilatata N. ab E. / B. microphylla. / Hab. in rupibus madidis collinum las Lomas del Alcornoque prope/MedinaSidonia. / Alt. 1000'. / Legi d. 22 Febr. 1845. /

b) Frullania dilatata N. ab E.

Etiqueta 1.- [impreso]: Herbarium Willkomm. / [m. Willkomm]: Frullania dilatata / Aragonia australis./[impreso]: Legi/ [m. Willkomm]: Loscos [impreso]: $18 /$

Etiqueta 2.- [m. Loscos]: Frullania dilatata símbolo masculino et femenino. / [m. Loscos]: 53 / Acaso Hepatica ? Planta abun / dante en los troncos y tambien / a veces sobre la tierra. / Jamas fructifica. /

Etiqueta 3.- [m. Willkomm]: Frullania dilatata N. ab E. / Hab. in Hispania. / Ex hb. d. Boutelou, 1844. I

c) Fr. nervosa Ml.

Etiqueta .- [impreso]: HERB. [m. Verdoorn]: Inst. Bot. Coimbra / [impreso]: $\mathrm{N}^{\mathrm{o}} /$ [m. Verdoorn]: Fr. nervosa Ml./ 3. 1929/ [impreso]: det. Verdoorn I

\section{BIBLIOGRAFÍA}

BONNER, C.E.B. -1962-78 Index hepaticarum Pars I-IX. Weinheim, Lehre or Vaduz, J. Cramer. (I, 1962; II, 1962; III, 1963; IV, 1963; V, 1965; VI, 1966; VII, Supplementum, H. Bischler, 1977; VIII, 1976; IX, Bischler, H. \& D. Lamy, 1978).

CASARES GIL, A. -1919- Flora Ibérica. Briófitas ( $1^{\text {a }}$ Parte). Hepáticas. Mus. Nac. Cien. Nat., 1 775. Madrid.

DÜLL, R. -1983- Distribution of the European and 
Macaronesian liverworts (Hepaticophytina). Bryol. Beitr., 2:115.

GROLLE, R. -1976- Lebermoose Europas. Feddes Repertorium. 87: 171-279 1981 Miscellanea Hepaticologica. Journ. Hattori Bot. Lab., 49:8592.

GROLLE, R. -1983a- Hepatics of Europe including the Azores: an annotated list of species, with synonyms from the recent literarure. J. Bryol., 12:403-456.

GROLLE, R. -1983b- Nomina Generica Hepaticarum; references, types and synonymies. Acta Bot. Fennica, 121:1-62.

HUSNOT, T. -1922- Hepaticologia gallica. Ed. 2. Cahan.

JOVET-AST, S. -1983- Taxons rares on critiques des genre Riccia, Cryptogamie, Bryol. Lichenol., 4 (4):315-333.

JOVET-AST, S. -1986- Les Riccia de la Région Mediterranéenne. Cryptogamie. Bryol. Lichénol., 7 (3):287-431.

MACVICAR, S.M. -1971- The student's handbook of British Hepatics. ( $2^{\mathrm{a}}$ ed.). London.

MÜLLER, K. -1906-16 Die Lebermoose. Leipzig.

SCHUSTER, R.M. -1969-80- The Hepaticae and Anthocerotae of North America. 4 Vols. New York and London.
SCHUSTER, R.M. -1983- New Manual of Bryology, 2 Vols. Hattori Bot. Lab. p. 1295 .Nichinan.

SMITH, A.J.E. -1990- The liverworts of Britain and Ireland. Cambridge University Press. Cambridge.

STEFANI, F. -1906-17 Species hepaticarum. 5 Vols. Lyon.

WILLKOMM, H.M. -1844-46 Botanische Berichte aus Spanien. Bot. Zeit. 2 (45):769-772, 1844a. 2 (45): 836-841, 1844b. 3 (18):29-302, 1845a. 3 (45):737-744, 1845b. 4 (34):577-588, 1846.

WILLKOMM, H.M. -1850- Vegetaetionsskizzen aus Spanien und Portugal. Bot. Zeit. 8, (27):505514.

Aceptado para su publicación en Julio de 1992

Dirección de los autores. Departamento de Biología Vegetal (Botánica). Facultad de Biología. Universidad de Santiago de Compostela. España. 